\title{
Absence of color-selectivity in Duncker-type induced visual movement
}

\author{
R. H. DAY and R. G. DICKINSON \\ Monash University, Clayton, Victoria 3168, A ustralia
}

\begin{abstract}
Using a stationary target and moving field, both consisting of gratings of vertical light and dark bars, Over and Lovegrove (1973) reported that, with monoptic viewing, induced target movement is weaker when the light bars of the two components are different in color. This reduction did not occur for dichoptic viewing, for which the aftereffect was almost negligible. Six experiments are described. The effect of different colors was not confirmed, using a stationary point and moving frame or stationary and moving gratings. Reduced effects for different colors and greatly reduced effects for dichoptic viewing occurred only when there was a stationary boundary to the moving bars of the field grating, as in Over and Lovegrove's experiment. It is concluded that the effect studied by Over and Lovegrove is not the classical induced movement described by Duncker (1929/1938) but one due to periodic coincidence and noncoincidence of moving and stationary bars in grating patterns. This effect is absent when target and field bars are rendered more distinguishable by different colors.
\end{abstract}

Induced visual movement, also called simultaneous motion contrast, is the apparent movement of a stationary element in the opposite direction to the real movement of another element in an otherwise featureless field. The effect is frequently demonstrated by means of a stationary point enclosed within a rectangular moving frame, but it occurs with a variety of stimulus patterns and movements (Duncker, 1929/ 1938; Wallach, 1959). Over and Lovegrove (1973) used a small, circular, stationary target centered in a larger circular field. Both target and field consisted of light and dark bars, with those of the latter moving laterally. When the light bars of target and field were of the same wavelength composition, induced movement, as indexed by manually tracked displacement, was markedly greater than when the two elements were different in composition. However, this difference in induced movement occurred only when target and field were presented to the same eye (monoptic presentation), not when the target was presented to one eye and the field to the other (dichoptic presentation).

Although Over and Lovegrove (1973) refer to the effect with grating patterns as simultaneous motion contrast, the conditions for its occurrence are superficially the same as those for induced movement described by Brosgole (1968), Duncker (1929/1938), and Wallach (1959). The stationary target appeared to move when the bars of the target actually moved. It will be argued below that the apparent movement

Requests for reprints should be addressed to Professor R. H. Day, Department of Psychology, Monash University, Clayton, Victoria 3168, Australia. This work was supported by Grant A65/15940 from the Australian Research Grants Committee. of a stationary grating target surrounded by a moving grating with a stationary boundary is a special effect which is attributable to the periodic coincidence and noncoincidence of the bars of target and field.

The rationale for Over and Lovegrove's (1973) experiment derived from recent studies of the visual motion aftereffect, the apparent motion of a stationary pattern in the opposite direction to its prior real movement. It had earlier been shown that with patterns consisting of light and dark bars the aftereffect is greater when the light bars are the same in wavelength composition (referred to hereinafter as color) during the moving (inducing) and stationary (test) phase than when they are different, providing that the pattern is viewed with the same eye during both phases. The difference in the strength of the aftereffect due to different color combinations does not occur when the pattern is viewed with one eye during the inducing phase and with the other in the test phase (Lovegrove, Over, \& Broese, 1972). These data suggested that detectors tuned to color and motion receive exclusively monocular inputs, whereas motion detectors that can be excited binocularly are insensitive to color. The point of Over and Lovegrove's experiment was to establish whether induced movement was also color specific. They interpreted the positive outcome as indicating that, like the visual motion aftereffect, induced movement results from inhibitory interactions between feature detectors with similar response characteristics. Such color sensitivity implies that inhibition occurs only between detectors that are tuned in the same way to motion and color.

There is another feature of the data reported by Over and Lovegrove (1973), which, while it has an important bearing on an explanation of induced 
movement, did not receive comment. It is that with dichoptic presentation of target and field induced movement was very markedly reduced. Its strength was only about $8 \%-15 \%$ of that for monoptic viewing. The graphed results show that the total displacement during a 15 -sec viewing period was only a few millimeters, suggesting that the effect was almost negligible. The fact that induced movement was marked with monoptic viewing but virtually absent with dichoptic viewing indicates that it might be largely or wholly subcortical in origin (Julesz, 1971).

Two considerations suggested that these important observations and conclusions concerning the basis of induced movement should be reexamined. Firstly, although there was no difference in induced movement between same and different colors for target and field in dichoptic viewing, the effect overall was so slight as to render this observation questionable. Secondly, informal observations with a stationary point and moving frame, the classical arrangement for demonstrating induced movement, did not confirm Over and Lovegrove's results for either the effects of wavelength or viewing mode. This suggested that the data which they reported might be confined to particular stimulus conditions.

Six experiments are described here. The first was designed to establish whether the effects of color combinations of target and field and of monoptic and dichoptic viewing reported by Over and Lovegrove (1973) for grating patterns holds for a display consisting of a stationary point and an enclosing, moving frame (Figure 1). With the negative outcome of this experiment, the second was designed to reexamine the issue with a target and field consisting of gratings (Figure 1). Since the data were again largely negative, Experiments 3 to 6 were con-

\section{EXPERIMENT I}

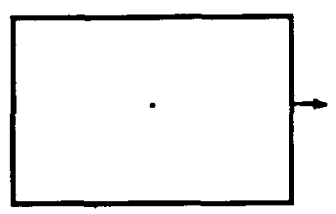

EXPERIMENTS III \&

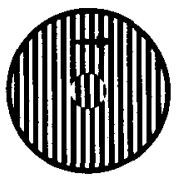

EXPERIMENT II

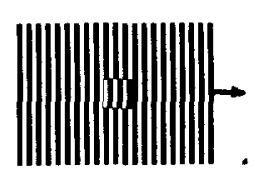

EXPERIMENTS $\mathbb{Z} \& \mathbb{Z}$

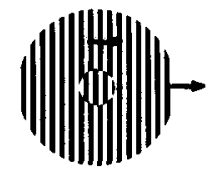

Figure 1. Displays used in the six experiments. In all displays, the surrounded element was always stationary but appeared to move. The parts of the surrounding fields which moved physically are shown by arrows. In the displays for Experiments 3 and 5 , the outer bars moved in a stationary aperture, indicated by the circular border. In Experiments 4 and 6, the entire field moved. ducted to ascertain the basis of the marked discrepancies between the results of Experiments 1 and 2 , on the one hand, and those reported by Over and Lovegrove, on the other, using the stimulus displays shown in Figure 1.

\section{EXPERIMENT 1}

The finding that induced movement with gratings is weaker in monoptic presentation when the colors of target and field are different has been interpreted by Over and Lovegrove (1973) as indicating that the effect is attributable to inhibitory interaction between feature-detectors with similar response characteristics. If this interpretation is generally valid, it is reasonable to expect that the results would hold for different stimulus patterns.exhibiting induced movement. The purpose of the first experiment, therefore, was to establish whether induced movement of a stationary point in a moving frame is reduced in monoptic presentation ${ }^{1}$ but unaffected in dichoptic presentation when point and frame are different in wavelength.

\section{Subjects \\ Method \\ There were 24 paid subjects, 13 men and 11 women, all of whom were undergraduate or graduate students.}

\section{Apparatus}

The main features of the apparatus have already been described (Day, Dickinson, \& Forster, 1976). The stimulus array (Figure 1) consisted of a small, square target which was always stationary and straight ahead of the subject at eye level, and a coplanar, rectangular frame which enclosed it (Figure 1). The viewing distance, which was controlled by a chinrest, was $257 \mathrm{~cm}$. The target was $.5 \mathrm{~cm}$ square (visual angle: $.11^{\circ}$ ). The border of the frame was $.5 \mathrm{~cm}\left(.11^{\circ}\right)$ wide. The frame was $30 \mathrm{~cm}\left(6.65^{\circ}\right)$ horizontally and $20 \mathrm{~cm}\left(4.43^{\circ}\right)$ vertically in outside dimensions. It could be moved horizontally in either direction at $.75^{\circ}$ and $.5^{\circ} / \mathrm{sec}$ by a motor operating through a gear box and a rack and pinion linkage.

The colors of target and frame were controlled by separate Kodak Wratten filters and dichoptic and monoptic presentation of the two elements by crossed and uncrossed polarizing filters. Kodak Wratten filters Nos. 26 (red) and 55 (green) were arranged to give color combinations of green-green (GG), green-red (GR), red-red (RR), and red-green (RG) of the target and frame, respectively. Polarizing filters were arranged in the display and in tightly fitting goggles so that the target and frame were presented either monoptically or dichoptically. The light source for target and field were appropriately arranged incandescent pearl lamps in a lightproof housing in back of the slides on which target and field were located. The luminance of both elements was adjusted to $1 \mathrm{~cd} / \mathrm{m}^{2}$.

Black draw-curtains in front of the stimulus array prevented any view of it when the subject entered the laboratory or between trials when the room lights were on. During a trial, only the sharply defined target and frame were visible in the otherwise dark laboratory.

A manual tracking device which had proved satisfactory earlier in recording both a visual movement aftereffect (Day \& Strelow, 1971; Strelow \& Day, 1971) and induced visual movement (Day, Dickinson, \& Forster, 1976; Over \& Lovegrove, 1973) was used throughout. A carriage mounted above a moving chart $(.70 \mathrm{~cm} / \mathrm{sec})$ was moved by the subject from side to side at right angles to 
chart movement to match the direction and speed of induced movement. A permanent record of this manual response was traced on the chart. The apparent velocity of induced movement was derived from the trace and served as the index of the strength of induced movement.

\section{Procedure}

Subjects were allocated to two groups of 12 . For one group, the two stimulus elements were presented dichoptically during all trials, and for the other, monoptically. During monoptic trials, the subjects viewed the display using both eyes.' Prior to the first trial standard, taped instructions were played to the subject and questions encouraged and answered. In this and all subsequent experiments, the subjects were instructed to observe the stationary target throughout a trial. Subjects in the dichoptic presentation group were asked to make certain before each trial that the spatial relationship between target and frame was the same as in monoptic view.

A trial consisted of manual tracking of the physically stationary target as it appeared to move from one side of the frame to the other. For the higher frame speed, the duration of a trial was $8.9 \mathrm{sec}$, and for the lower speed, $13.3 \mathrm{sec}$. Each subject underwent eight trials, two under each of the four target-frame color combination conditions: GG, GR, RR, and RG. The speed of frame movement for one trial under each condition was $.75^{\circ} / \mathrm{sec}$, and for the other, $-5^{\circ} / \mathrm{sec}$. The order of the eight trials was differently randomized for each subject. The two randomly distributed frame speeds were included to check that subjects were responding to the apparent speed of the target and not moving the hand independently of apparent velocity. For half the subjects in each group, the frame was moved during all eight trials from left to right so that the target appeared to move from right to left, and for the other half, it was moved from right to left.

The score for each subject under each of the color combinations and speed and viewing conditions was the average tracked velocity of induced target movement calculated from the slope of the recorded trace.

\section{Results and Discussion}

The mean tracked velocities of induced target movement for the color combinations and viewing conditions are shown separately for the two speeds $\left(.75^{\circ}\right.$ and $\left..5^{\circ} / \mathrm{sec}\right)$ in Figure 2. An analysis of variance has shown that the only main effect to achieve significance was target speed $[F(1,22)=320.05, p<.01]$. Contrary to expectation from Over and Lovegrove's
(1973) experiment, the speed of induced movement did not depend on the interaction between frame and target color $[F(1,22)=.16$, n.s.]. However, as would be predicted from their results, neither the color of the frame nor that of the target significantly affected induced movement $[\mathrm{F}(1,22)=.01$, n.s.; $\mathrm{F}(1,22)=$ $1.08, \mathrm{p}>.05]$. While there was no difference in induced movement between monoptic and dichoptic viewing $[F(1,22)=1.21, p>.05]$, the interaction between mode of viewing and target speed was significant $[\mathrm{F}(1,22)=5.83, \mathrm{p}<.025]$.

The results reported by Over and Lovegrove (1973), using a target and field consisting of grating patterns, have not been sustained with a stimulus array consisting of a stationary point enclosed in a moving frame. Although the apparent speed of induced movement varied significantly with the speed of real movement of the frame, it showed no reduction when the colors of target and frame were different. Moreover, the difference between monoptic and dichoptic viewing was slight and achieved significance only at the greater of the two speeds.

\section{EXPERIMENT 2}

The question which now arises is whether the inconsistency between the results for the two experiments was due to the different displays. It is conceivable that the effect of the color combination of target and field during monoptic viewing is restricted to grating patterns. The second experiment was designed to resolve this issue. Except for the stimulus array and some minor modifications to the apparatus necessary to accommodate the new display, the design and procedure were the same as in the first experiment.

\section{Subjects}

\section{Method}

There were 12 new, paid subjects, 6 men and 6 women, all undergraduates.

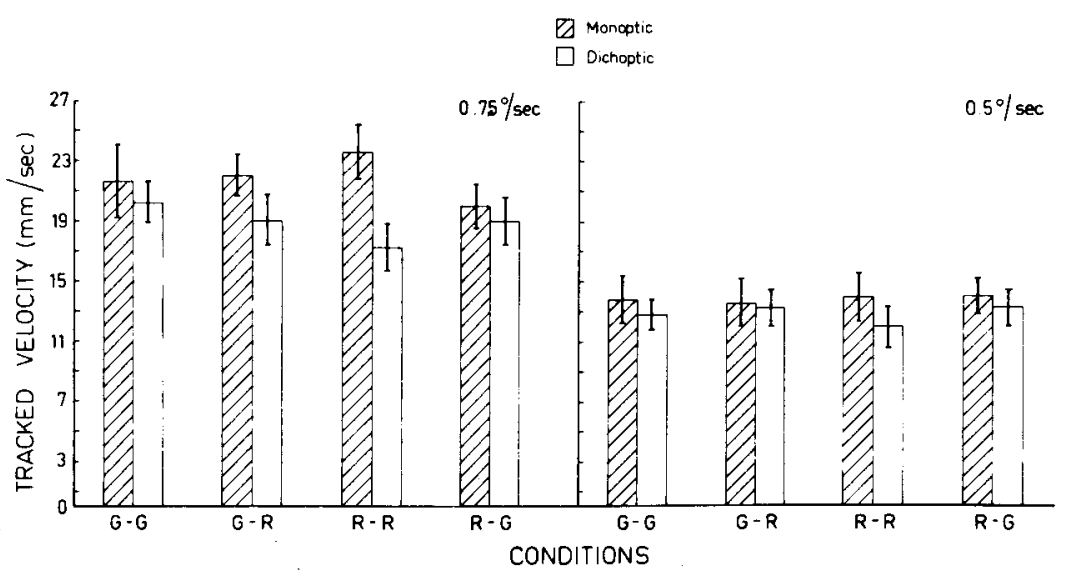

Figure 2. Mean tracked velocities of stationary point for four color combinations of point and frame, monoptic and dichoptic presentation, and two frame speeds (Experiment 1). Standard errors of the means are also shown as vertical lines. 
Apparatus

The display shown in Figure 1 consisted of a stationary target, $3 \mathrm{~cm}\left(.66^{\circ}\right)$ square, and a rectangular surrounding field, $21 \mathrm{~cm}$ $\left(4.65^{\circ}\right)$ wide and $15 \mathrm{~cm}\left(3.32^{\circ}\right)$ high. Target and field consisted of vertical light and dark bars, each $.5 \mathrm{~cm}\left(.11^{\circ}\right)$ wide. The light bars of each element could be made red or green by means of the same Wratten filters (Kodak Nos. 26 and 55) used in the first experiment. Dichoptic and monoptic presentation of target and field were again controlled by crossed and uncrossed polarizing filters. Since the target in this experiment was superimposed on (rather than surrounded by) the field, it was necessary to introduce a half-silvered mirror between display and subject. The mirror was mounted horizontally but tilted forward at $45^{\circ}$ with the top edge nearer the subject. The target housing with an incandescent, pearl lamp was located below the mirror so that the target itself was reflected from the front surface. The field housing was mounted behind so that the field was transmitted through the mirror. The luminance of target and field was adjusted to equality at $.08 \mathrm{~cd} / \mathrm{m}^{2}$. The reduction in luminance relative to that in Experiment 1 was necessary in order to prevent light scatter in the laboratory with consequent illumination of features other than the stimulus pattern. ${ }^{2}$ All other features of the apparatus, including frame speeds, viewing distance, and tracking device, were the same as in the first experiment.

\section{Procedure}

The design, procedure, and instructions for the experiment were the same as in Experiment 1 . However, since the moving field was less wide than in the first experiment, the duration of a trial during which the subject tracked induced movement was $6.2 \mathrm{sec}$ for the higher speed of the field and 9.3 for the lower. The 12 subjects were allocated to two groups of 6 . with an equal number of men and women in each. One group viewed the display monoptically for the four color combinations, and the other viewed it dichoptically, with the direction of frame movement for all trials alternating between subjects.

\section{Results and Discussion}

Mean tracked velocities for the eight conditions, together with standard errors, are shown in Figure 3. An analysis of variance similar to that for the first experiment showed that, while there was a significant main effect due to frame speed $[F(1,10)=11.88$, $\mathrm{p}<.01]$, neither the interaction between surround and target color $[\mathrm{F}(1,10)=.56$, n.s.] nor the main effects due to surround and target colors $[F(1,10)=$ $1.67, \mathrm{p}>.05$, and $\mathrm{F}(1,10)=1.62, \mathrm{p}>.05$, respectively] were significant. The difference between monoptic and dichoptic viewing also failed to achieve significant, $F(1,10)=1.17, p>.05$. No other interaction achieved significance.

In summary, with a grating display similar to that in Over and Lovegrove's (1973) experiment, no difference in induced movement was found between same and different colors in target and field and between monoptic and dichoptic presentation of these two parts of the display.

\section{EXPERIMENTS 3 AND 4}

In Experiments 1 and 2, the entire field, frame or grating, moved laterally as depicted in Figure 1. However, in Over and Lovegrove's (1973) experiment, the field grating moved within a stationary circular boundary formed by an aperture. The aperture was placed between subject and oscilloscope face presumably to mask the edges of the tube. While on first consideration it seems unlikely that the presence of a stationary boundary encircling the moving bars could account for the marked discrepancy between the two sets of data, it was nevertheless considered worthwhile to explore the possibility. Therefore, the purpose of Experiment 3 was to investigate the effect of same and different targetfield color combinations with a display as similar as possible to that used by Over and Lovegrove. Subjects viewed the display monocularly throughout. Since a difference was found between the two types of color combination, Experiment 4 was carried out to establish whether the difference was eliminated (as in Experiment 2) when the entire circular field moved.

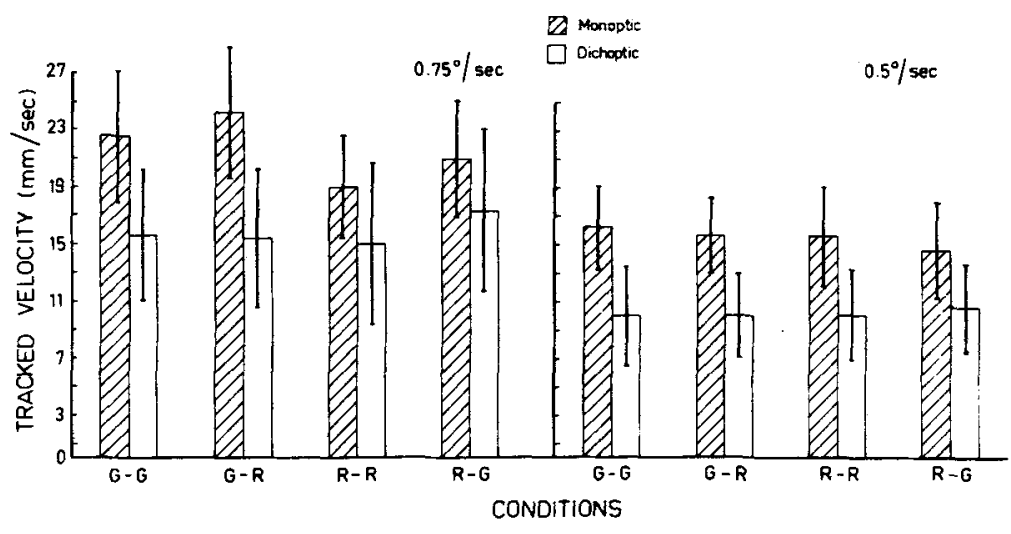

Figure 3. Mean tracked velocities of stationary grating target for four color combinations of target and field gratings, monoptic and dichoptic presentation, and two field speeds (Experiment 2). Standard errors of the means are shown as vertical lines. 


\section{Experiment 3}

\section{Method}

Subjects. There were 24 new, paid subjects, 11 men and 13 women, all undergraduate or graduate volunteers.

Apparatus. The display was modified to include a stationary, circular aperture, behind which the vertical bars of the grating pattern moved laterally and in which a circular, stationary gratingthe target-was centered (Figure 1). The viewing distance was altered to $287 \mathrm{~cm}$, at which $5 \mathrm{~cm}=1^{\circ}$ of visual angle. The aperture was $3^{\circ}$ in visual angle, the stationary target $.7^{\circ}$, and the light and dark bars $.1^{\circ}$. Thus, the spatial frequency was 5 cycles/deg. The bars moved right or left at $.4 \% \mathrm{sec}$. The color of the light bars was again controlled by Wratten filters Nos. 26 (red) and 55 (green). The luminance was $.05 \mathrm{~cd} / \mathrm{m}^{2}$.

Procedure. There were eight trials, two under each of the conditions, GG, GR, RR, and RG, with the order of conditions differently randomized for each subject. Each pair of trials under one condition was completed before the next was undertaken. The direction of frame movement for one trial of each pair was from right to left and for the other from left to right, with the initial direction alternating from subject to subject. Each trial was $15 \mathrm{sec}$ in duration and the right eye only was used throughout, as in Over and Lovegrove's (1973) experiment (see Footnote 1). The subjects were instructed always to observe the stationary target.

\section{Results}

The mean tracked velocities of induced movement of the target are shown with their standard errors in Figure 4. An analysis of variance showed that in this experiment the magnitude of induced movement did depend on the interaction between the field and target colors $[\mathrm{F}(1,23)=5.98, \mathrm{p}<.025]$ but that neither the surround color $[F(1,23)=.15]$ nor the test color $[F(1,23)=.70]$ were significant as main effects. Thus, with a stationary contour enclosing the field of moving bars, induced movement of the stationary target was stronger as indexed by tracked velocity when the colors of the bars of target and field were the same than when they were different.

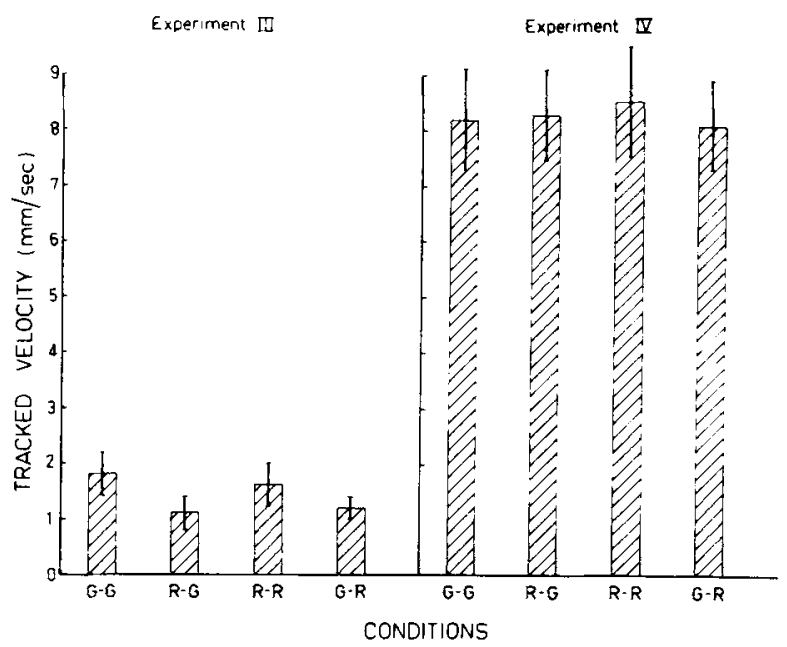

Figure 4. Mean tracked velocities for four color combinations of target and field gratings with monoptic presentation and stationary field boundary (Experiment 3 ) and no stationary field boundary (Experiment 4). Standard errors of the means are shown as vertical lines.
It can be noted that if, in Over and Lovegrove's (1973) experiment, the mean displacements during $15 \mathrm{sec}$ are converted to velocities there is a very close correspondence between their results and those for Experiment 3. For conditions GG, RG, RR, and GR, Over and Lovegrove's means calculated from their graphed displacements were about 1.96, 1.00, 2.06, and $1.10 \mathrm{~mm} / \mathrm{sec}$. In Experiment 3, the results for the same conditions were $1.75,1.16,1.61$, and $1.25 \mathrm{~mm} / \mathrm{sec}$. If allowance is made for the slight difference in inducing velocities $(.5$ and $.4 \mathrm{deg} / \mathrm{sec})$, the present results can be taken as closely corresponding with and confirming those of Over and Lovegrove.

\section{Method}

\section{Experiment 4}

Subjects. Another group of 24 paid, undergraduate volunteer subjects, 12 males and 12 females, took part.

Apparatus. The sole difference between the apparatus for this experiment and that for Experiment 3 was the absence of the stationary contour formed by the aperture around the field. The entire circular field of dark and light bars moved right or left during the 15-sec trial, as in Experiments 1 and 2. The display is depicted in Figure 1.

Procedure. The procedure was the same as for Experiment 3.

\section{Results}

The means of tracked target velocities and their standard errors are also shown in Figure 4. An analysis of variance has shown that in this experiment induced movement did not depend on the interaction between the field and target color $[F(1,23)=.13$, n.s.] and that, again, neither the field color $[\mathrm{F}(1,23)$ $=.05$, n.s.] nor target color $[\mathrm{F}(1,23)=1.45, \mathrm{p}>.05]$ were significant variables.

\section{Discussion}

The data from Experiments 3 and 4 can be summarized as follows. Relative to the condition in which target and field gratings were the same color, induced movement of a stationary target grating of one color was reduced when a moving field grating of another color was enclosed by a stationary aperture. However, this reduction did not occur when the aperture was removed so that the entire field grating moved. Thus the data reported by Over and Lovegrove were confirmed. An explanation of the difference between the two field arrangements is proposed in the general discussion below.

\section{EXPERIMENTS 5 AND 6}

Over and Lovegrove (1973) found that with grating patterns induced movement is considerably weaker for dichoptic viewing of target and field than it is for monoptic. The results from Experiments 1 and 2 did not accord with this finding. In Experiment 1, a difference between the two viewing modes occurred 
only at one of the two frame speeds and was relatively slight. In Experiment 2, there was no difference at either frame speed. The purpose of Experiments 5 and 6 was to establish whether a difference in induced movement between monoptic and dichoptic viewing with a grating display occurs when there is a stationary boundary formed by an aperture to the field of moving elements. In Experiment 5, a stationary boundary was present, and in Experiment 6, the whole field moved, as shown in Figure 1. The two displays were the same in all other respects.

\section{Experiment 5}

\section{Method}

Subjects. There were 12 new, paid subjects, 4 men and 8 women, recruited from the same population as in the earlier experiments.

Apparatus. The apparatus was the same as for Experiment 3, with a stationary, circular boundary to the field formed by an aperture in a dark screen (Figure 1). The color of the field was controlled throughout by Wratten filter No. 55 so that both elements were green.

Procedure. Each subject underwent eight trials, two pairs of trials with monoptic (M) presentation and two pairs with dichoptic (D) presentation. The order of the four pairs for half the subjects was DMMD and that for the other half MDDM. The direction of frame movement for one trial of each pair was from right to left and that for the other from left to right, with the initial direction of each pair alternating from subject to subject. During monoptic trials, the subjects viewed the display with the right eye only.

\section{Results}

The mean tracked velocities of induced movement for the monoptic and dichoptic conditions are shown, together with standard errors, in Figure 5. A onetailed $t$ test for correlated means has shown that the

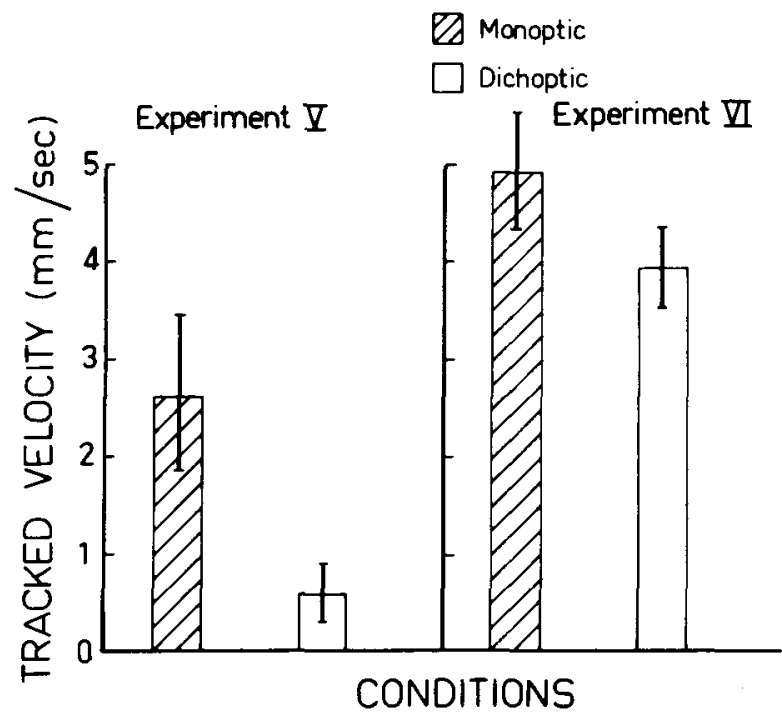

Figure 5. Mean tracked velocities for monoptic and dichoptic presentation of target and field gratings with stationary field boundary (Experiment 5) and without stationary field boundary (Experiment 6). Standard errors of the means are shown as vertical lines. difference between the means for monoptic and dichoptic viewing was significant, $\mathrm{t}(11)=2.98$, $\mathrm{p}<.01$, thus confirming Over and Lovegrove's finding.

\section{Experiment 6}

\section{Method}

Subjects. A group of 12 subjects, 6 men and 6 women, participated. They were recruited and paid in the same way as the earlier subjects.

Apparatus. The apparatus was the same as for Experiment 5, except for the stationary boundary to the field of moving bars. The display was arranged so that the entire circular field moved laterally, as in Experiments 1, 2, and 4, as shown in Figure 1.

Procedure. The procedure was the same as for Experiment 5.

\section{Results}

The mean tracked velocities for induced movement with monoptic and dichoptic viewing are shown with their standard errors in Figure 5. A one-tailed t test for correlated means has shown that there was a significant difference between the two means [t(11) $=1.90, \mathrm{p}<.05]$.

\section{Discussion}

In Experiment 5, induced movement with dichoptic viewing of target and field was slower than for monoptic viewing with a stationary boundary to the field of moving bars and in Experiment 6 in which the entire field moved. However, while in Experiment 5 the reduction was about $80 \%$ of the monoptic velocity, in Experiment 6 it was much less, about $20 \%$. That is, with a stationary boundary there was a very marked reduction in induced movement for dichoptic viewing, but without such a boundary the reduction was relatively small.

Because the actual speed of the field or field elements in Experiments 4 and 6 was the same, the difference in speed of induced movement for monoptic viewing between Experiments 4 (Figure 4) and 6 (Figure 5) calls for comment. It can be seen that while the mean tracked velocities in Experiment 4 were about $8.5 \mathrm{~mm} / \mathrm{sec}$ those in Experiment 6 were about $4-5 \mathrm{~mm} / \mathrm{sec}$, nearly half. This difference is probably attributable to the within-groups design and consequent interaction between conditions. Thus, the relatively slight effect in Experiment 6 for dichoptic presentation conceivably attenuated that for monoptic viewing. In Experiment 4, there was no such reduced effect for any of the conditions. The possibility of interactions between conditions with consequent establishment of "norms" of apparent velocity deserves closer investigation.

\section{GENERAL DISCUSSION}

The results of the six experiments can be summarized as follows. With a stimulus array consisting of a stationary point as target and a moving, enclosing, 
rectangular frame, induced movement of the point did not vary when the two were the same or different in color or when they were viewed monoptically or dichoptically (Experiment 1). With an array in which the stationary target and moving field consisted of vertical light and dark bars, essentially the same results obtained (Experiment 2). With similar circular grating targets and fields, induced movement for different color combinations of the two stimulus components was reduced for the condition in which there was a stationary boundary to the field of moving bars but not for that in which the whole field moved (Experiments 3 and 4). For the stationary contour condition, induced movement with dichoptic viewing was reduced by about $80 \%$ relative to monoptic viewing but by only about $20 \%$ when the entire field moved (Experiments 5 and 6 ).

These outcomes indicate that the results found by Over and Lovegrove (1973) for the effect of same and different colors between target and field are restricted to certain stimulus conditions. The results do not obtain in the classical situation involving movement of the entire field surrounding the stationary target. Thus, the general conclusion that induced movement results from inhibitory interaction between feature detectors with similar response characteristics based on color-selectivity in monoptic viewing must be rejected.

While none of the conclusions from Over and Lovegrove's (1973) study derived from the marked reduction in induced movement with dichoptic viewing, this result nevertheless suggests the involvement of subcortical processes. However, in the experiments reported here, such reduction when it occurred was much less than that found by Over and Lovegrove. A large reduction occurred only under the condition in which there was a stationary contour to the field of moving bars.

A basis for the results found for color combinations and dichoptic viewing with a stationary boundary to the field of moving bars is suggested by the overall mean velocities of induced movement with and without such a boundary and by informal observation of the quality of movement under the two conditions. The means in Figures 3 and 4 show that induced movement was considerably weaker when there was a stationary boundary to the moving bars than when the whole field moved. The mean velocity of movement with a stationary boundary in Experiment 3 was only about $17 \%$ of that in Experiment 4 without it (Figure 4, and in Experiment 5 about $35 \%$ of that in Experiment 6 (Figure 4). Informal observation revealed that when the entire field moved perceived movement of the target was a compelling effect, the target appearing to move smoothly and continuously in the manner described by Duncker (1929/1938) and Wallach (1959). With a stationary boundary around the bars, the effect was different. The target seemed to move jerkily, rather as if the bars were appearing and then disappearing behind those of the field. Apparent movement consisted of a number of such episodes of appearance and disappearance as the bars of the target changed from noncoincidence to coincidence with those of the field.

On the basis of relative velocity under the two conditions and the informal observations of the quality of apparent movement, it is suggested that the effect with vertical bars and a fixed boundary is not induced movement in the generally accepted sense. Rather, it is a series of "appearances" and "disappearances" as the target bars change from being out of phase (noncoincidence) to in phase (coincidence) with the field bars. The stationary boundary serves as a reference which prevents the smooth, continuous, gliding effect commonly associated with Duncker-type induced movement. It seems likely that the marked reduction in the effect with target and field bars of a different color is simply due to the greater distinguishability of the two. When the target bars are more distinguishable, they can be more easily related perceptually to the stationary contour which serves as a reference. In consequence, they seem hardly to move.

A slight reduction in induced movement with dichoptic viewing occurred for the higher frame speed in Experiment 2 and in Experiment 6. Although the means for dichoptic viewing were smaller than for monoptic in Experiment 1 and for the lower frame speed in Experiment 2, the reduction was not significant. However, the reduction in Over and Lovegrove's (1973) experiment and in Experiment 5, which was essentially similar, was very much greater. What is the basis of these reductions with dichoptic presentation of target and field?

At this stage, a definite answer to this question cannot be given. However, dichoptic presentation of two parts of a stimulus pattern usually gives rise to perceptual instability, one part frequently drifting and changing its spatial relationship with the other. If, as a result of such instability, the target were separated from the field, a reduction in induced movement could be expected. Separation between the two components would be of less consequence for the classical effect with a moving field, since induced movement occurs with quite large distances between target and field (Duncker, 1929/1938). For the effect due to target and field bars moving in and out of coincidence with each other, such perceptual separation between the two would virtually eliminate it. If the target and field are visually separated, no effect due to coincidence and noncoincidence between the vertical bars would be possible. It can be noted in support of this argument that Bassili and Farber's (1977) subjects experienced difficulty in maintaining 
target and field in their proper spatial relationship when the display was presented dichoptically, as in Experiments 5 and 6 . However, when steps were taken to stabilize the display using a haploscopic arrangement, induced movement readily occurred.

Whether or not this attempt to account for the difference between the results obtained by Over and Lovegrove (1973) and in the experiments reported here is valid, data from the latter indicate that an interpretation of induced movement in terms of inhibitory interactions between feature analyzers tuned to color and movement is premature. The results described here offer no encouragement at all for such an explanation.

\section{REFERENCES}

Bassili, J. N., \& Farber, J. M. Experiments on the locus of induced motion. Perception \& Psychophysics, 1977, 21, 157-161.

Brosgole, L. An analysis of induced motion. Acta Psychologica, 1968, 28, 1-44.

DAY, R. H., Dickinson, R. G., \& Forster, K. I. Induced subjectrelative movement: Persistence of apparent movement of a stationary point after removal of inducing stimulus. Perception \& Psychophysics, 1976, 19, 510-517.

DAY, R. H., \& StRelow, E. R. Reduction or disappearance of visual after effect of movement in the absence of a patterned surround. Nature, 1971, 230, 55-56.

DunCKer, K. Induced motion (1929). In W. D. Ellis (Ed.), A source book of Gestalt psychology. London: Paul, Trench \& Trubner, 1938.
Julesz, B. Foundations of cyclopean perception. Chicago: University of Chicago Press, 1971.

Lovegrove, W. J., Over, R., \& Broese, J. Color-selectivity in motion aftereffect. Nature, 1972, 238, 334-335.

OVER, R., \& Lovegrove, W. Color-selectivity in simultaneous motion contrast. Perception \& Psychophysics, 1973, 14, 445-448.

Strelow, E. R., \& DAY, R. H. After effect of visual movement: Storage in the absence of a patterned surround. Perception \& Psychophysics, 1971, 9, 485-486.

Wallach, H. Perception of motion. Scientific American, 1959, 201, 56-60.

\section{NOTES}

1. Dichoptic vision refers to presentation of one part of the stimulus pattern to the left eye and the other part to the right eye. However, monoptic vision can refer to presentation of the two parts to either one eye (monocular viewing) or both eyes (binocular viewing). Unless binocular summation occurs, there is no essential difference between these two. A control experiment using essentially the same pattern and procedures as in Experiment 1 , with eight subjects, showed that there is no difference in tracked velocity between monocular and binocular viewing, i.e., that there is no binocular summation of induced movement.

2. Another control experiment with eight subjects involving the same display as in Experiment 1 showed that the tracked velocity of a stationary target did not vary as the luminance of the display was varied in five steps between .02 and $1.89 \mathrm{~cd} / \mathrm{m}^{2}$. That is, induced movement remained stable with change in luminance.

(Received for publication November 15, 1976; revision accepted June 23, 1977.) 